\title{
UNA SESIÓN INNOVADORA DE TRABAJO DE LABORATORIO PARA ENSEÑAR PROCESO DE DATOS. SEGUNDO CURSO DE ESTUDIOS DE FÍSICA A NIVEL UNIVERSITARIO
}

\author{
FONDÈRE, F, y SÉRÉ, M.G. \\ Grupo DidaScO. Universidad de París XI Bat 336. 91405 Orsay (Francia).
}

\section{SUMMARY}

An innovative reflection about measurement is proposed through Franck and Hertz experiment for second year Universify students. We present a sequence of laboratory work during which the students have the opportunity of deepening notions like dispersion, error and uncertainty, accuracy and precision, and some concepts of statistics. We describe how students are led to develop personal comments on their own results.

\section{INTRODUCCIÓN}

Este artículo presenta una sesión innovadora de trabajo de laboratorio, siguiendo, y teniendo en cuenta, ciertas investigaciones sobre análisis estadístico de datos, a nivel de los estudios universitarios de física. Obviamente, en cualquier actividad profesional relacionada con la física, los conocimientos en cuanto a los métodos de modida desempeñan un papel importante. Recientemente, al menos dos factores han propiciado una mayor reflexión acerca de las mediciones y del análisis estadístico de datos entre los investigadores e ingenieros: hoy en dia, hay cada vez más dispositivos automáticos especiales y resulta cada vez más fácil obtener muchas medidas; además, la presencia de ordenadores en los laboratorios facilita enormemente el proceso estadístico de datos. Existen diferentes métodos disponibles (Jaech, 1992) y merece la pena, al mismo tiempo que se plantea si resultan relevantes, cómo y a qué nivel, introducir a los estudiantes en estos métodos y en los correspondientes conceptos. Pcnsamos que ha llegado el momento de introducir a los estudiantes universitarios en esta nueva forma de abordar los experimentos; es decir, repetir las medidas y procesar los datos con métodos estadísticos. Ello parece constituir el objetivo inmediato de la enseñanza práctica en la universidad, con la intención de proporcionar a los estudiantes la capacidad de criterio para decidir si realizan varias medidas o no.

Pocos han sido los estudios de investigación dedicados a los conocimientos sobre medidas y análisis de datos, y casi nunca a nivel de estudios universitarios. Algunos están dedicados a los estudiantes más jóvenes (Bottin, Hot y Martinand, 1983; Coelho, 1993; Lubben y Millar, 1996), y constituyeron un importante incentivo para nuestro equipo a emprender una investigación acerca de las concepciones de los estudiantes, al cmpezar la universidad, y acerca de los cambios conceptuales necesarios para entender el proceso de medida y, especialmente, para utilizar correctamente el análisis estadístico de errores (Séré, Journeaux y Larcher, 1993; Fondère, 1993; Larcher, Séré y Joumeaux, 1993; Sćré, 1995). Los principales métodos utilizados fueron la observación directa en clase durante el trabajo de laboratorio (sobre 
temas seleccionados del trabajo de laboratorio) y las entrevistas individuales. La sesión innovadora de trabajo de laburatorio, objeto de estc informe, debe considerarse como una aplicación directa de esta investigación.

En la primera parte describimos brevemente la sesión en sí misma, que consiste en realizar el experimento de Franck y Hertz, destacando las oportunidades que se ofrecen a los estudiantes para profundizar cn la práctica de la medición y del proceso de datos. En la segunda parte consideraremos algunos resultados de la investigación. Describiremos algunas fases de la sesión quue proporcionan la oportunidad para superar las concepciones erróneas señaladas y para generar una comprensión mejorada de la medida, del procesamientos de datos y del análisis estadístico de errores.

\section{LA SESIÓN INNOVADORA DE TRABAJO DE LABORA'TORIO: EL EXPERIMENTO DE FRANCK Y HER'TZ}

Históricamente, este experimento, realizado en 1914 utilizando un tubo calentado que contiene un poco de vapor de mercurio, permitió comprobar la discontinutdad de los niveles de energía atómica y determinar el valor del intervalo $V_{0}$ entre el nivel de tierra y el primer nivel de chergía posible.

Este experimento es el tema de la sesión de laboratorio propuesta a los estudiantes de segundo curso universitario (en general entre 19 y 21 años), con el objetivo de que se familiaricen con la teoría de los niveles de energía. El tiempo se limita a cuatro horas. Lua distintas tareas que los estudiantes deben realizar son las siguientes:

- Como en todo trabajo de laboratorio, los estudiantes deben estudiar el libro de texto en el que se expone el proceso expcrimental que habrán de seguir en el laboratorio.

Figura 1

Malcriales para el cxperimento de Franck y Hertz

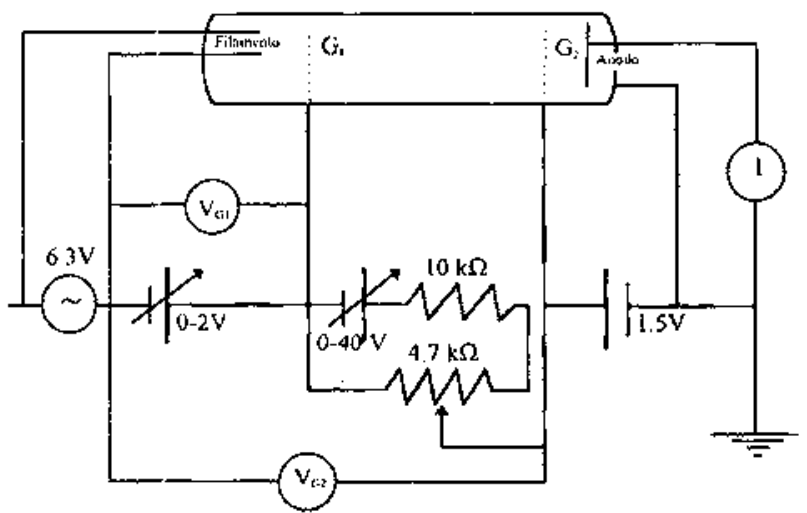

- Como preparación para el tratamiento de datos, deberán resolver dos ejercicios en los que intervicnen series de números y conceptos como: media, desviación estándar, distribuciones, distribución especial de Gauss, muestra y población, etc.

- El trabajo experimental, en sí mismo, consiste en registrar las medidas necesarias para trazar curvas que muestren la variación de intensidad I en el circuito externo (medida mediante un nano-amperímetro), como función del potencial de la rejilla de acelcración $V_{G:}$ (Fig. 1).

Los estudiantes deberán elegir, por sí mismos, algunos parámetros, por ejemplo, la temperatura del horno (lo cual modifica el número de átomos de mercurio contenidos en el vapor) y el potencial de la rejilla $V_{G t}$ (lo cual modifica el flujo de electrones acelerados). La figura 2 presenta las curvas típicas $I=f\left(V_{c 2}\right)$ que los estudiantes deberán trazar. Estas curvas dependen del parámetro $V_{G H}$, así como de la temperatura $T$. Los estudiantes deberán mostrar en qué forma influyen estos dos parámetros sobre $I$.

- A continuación, tendrán que interpretar las curvas de la figura 2 cualitativamente. Si la elección de los parámetros es correcta, bastarán tres curvas para mostrar la invarianza de las posiciones de los valores extremos de la intensidad $l$. Los estudiantes podrán obtener de cada curva un valor $V_{o}$ (de aquí se puede calcular la energía del estado excitado $e V_{o}$ siendo $e$ la carga del electrón) y la incertidumbre de estc valor.

- Finalmente, los estudiantes tendrán que analizar los datos con el fin de comparar el valor obtenido mediante este método con el valor esperado. Fs fundamentalmente durante este último paso cuando se ponde de manifiesto la importancia del análisis estadístico de errores.

- Los estudiantes deberán escribir un informe con los principales resultados experimentales, comentándolos y comparando los valores obtenidos con los esperados.

\section{ALGUNOS RESULTADOS DE LA INVES. TIGACION: LAS CONCEPCIONES DE LOS ESTUDIANTES Y EL CORRESPONDIEN'TE CAMBIO CONCEP'TUAL NECESARIOPARA ENTENDER EL ANÁLISIS ESTADISTICO DE DATOS}

Entre los resultados de la investigación mencionados anteriormente, seleccionamos algunos puntos aplicables a la sesión presentada.

2.1. Los estudiantes se muestran generalmente reacios a realizar varias medidas $y$, si repiten dichas medidas, intentan averiguar cuál es el valor correcto

Generalmente, al inicio de la universidad, los estudiantes no son verdaderamente conscientes de la posibilidad 
Figura 2

Variación de la intensidad $/$ con el potencial de la rejilla de aceleracion $V_{c z}$ a distintas temperaturas.
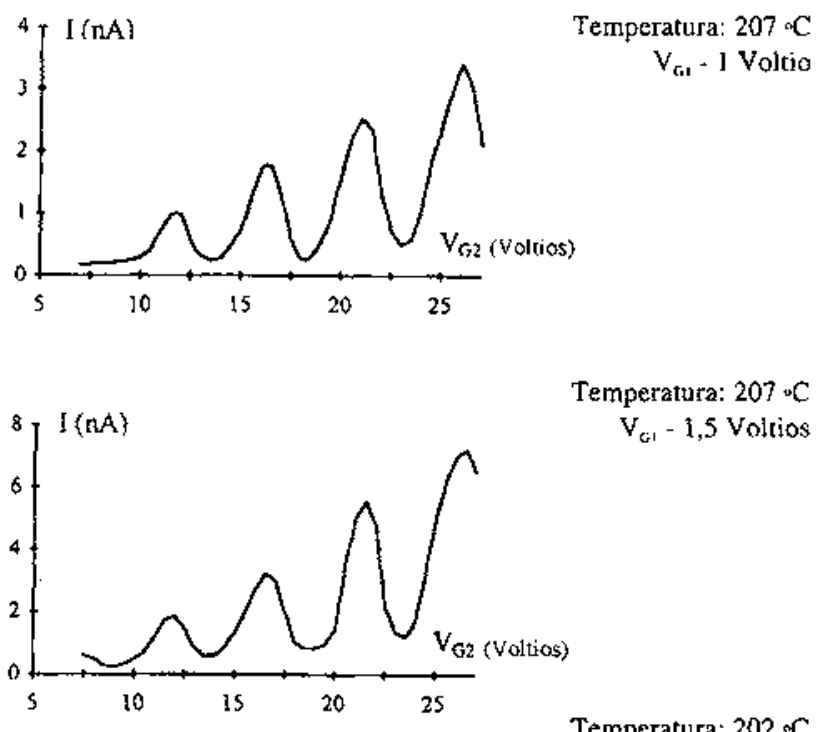

Temperatura: $207 \circ \mathrm{C}$ $V_{G 1}-1,5$ Voltios

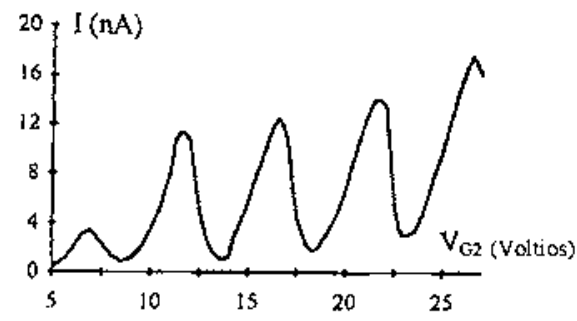

Temperatura: $202 \times \mathrm{C}$ $\mathrm{V}_{\mathrm{c} 1}-1,5$ voltios

de hacer varias medidas para mejorar la precisión. Se les ha enseñado a hacer solamente una medida y a acompanarla con un intervalo que es, en cierto modo, un cintervalo de certidumbre» o un «intervalo 100\%». En algunos casos, no resulta sencillo evaluar este tipo de intervalo, especialmente cuando resulta difícil decidir si es necesario añadir muchos elementos a la incertidumbre, lo cual, a menudo, conduce a un valor global excesivamente aito $y$, consiguientemente, poco realista.

Una aiternativa, siempre que sea posible desde un punto de vista práctico, consiste en indicar que varias medidas proporcionan más información que una, y que conviene hacer varias medidas, puesto que, en general, hay que tener en cuenta que ampljar el número de medidas mejora la precisión (aunque en absoluto la exactitud). No obstante, este punto de vista está lejos de las concepciones de los estudiantes. Al encarar el hecho de que las medidas son distintas, su tendencia es a buscar el valor único "correcto» de los del conjunto de medidas. Algunos estudiantes rechazan incluso la solución que consis. te en tomar la media cuando ésta no es igual a uno de los valores de la serie.

\subsection{Un equívoco conceptual acerca de la desviación estándar de las series de medidas}

En su inmensa mayoría, los estudiantes tienen la impresión de que, cuando aumenta el número $N$ de medidas, la media es más bien constante, pero piensan que la desviación estándar es menor. (Véase en 3 .I una explicación cualitativa a este hecho dada a los estudiantes en un ejercicio escrito.) No solamente son los estudiantes sino también los profesores los que tienen esta concepción etrónea. Una joven profe. sora entrevistada sobre este tema predijo correctamente que:

"Cuando crece $N$, nada cambia respecto a la media."

Y, en cambio, se equivocó acerca de la desviación estándar:

«Cuanto mayor es $N$, menor es la desviación estándar.»

«Cuantas menos medidas tengamos, más lejos estará la desviación estándar de la de la población.»

Estas respuestas en una joven profesora dan una idea de las dificultades a las que los estudiantes se enfrentan en situaciones similares.

\subsection{No se reconoce Ia intervención del azar: confusión entre errores sistemáticos y errores aleatorios}

Queda de manifiesto que la intervención de la condición de impredecible y del azar en física resulta sorprendente a los estudiantes. Pero deberán comprender que cada medida puede quedar modificada por errores aleatorios y que la estadística demuestra que la distribución de un número infinito de medidas tiene un carácter predecible: es una distribución de Gauss, bajo ciertas condiciones que son las del teorema del límite central. Entre estas condiciones, la más crítica es, probablemente, la independencia de las medidas acumuladas. Esta condición se cumple para los diferentes valores de $V_{0}$ obtenidos por cualquiera de los equipos de dos estudiantes $\mathrm{y}$, a fortiori, por un equipo diferente.

Al no tener una idea de la posibilidad del azar, el error aleatorio es una noción de la que no disponen los estudiantes. Además, algunos de ellos asignan un sentido corriente y común al término «sistemático», interpretándolo como «inevitable», ya que se sienten extraños y son muy conscientes de la dificultad de obtener un valor correcto, que, para ellos, es único. Del mismo modo, no distinguen realmente entre exactitud y precisión. Y esta dificultad se observa también en algunos profesores.

Se podría argumentar que los equívocos conceptuales de este tipo se deben al hecho de que los errores sistemáticos o aleatorios, asf como la exactitud o la precisión, adquieren su pleno sentido y, consecuentemente, resultan más fáciles de definir y de distinguir en el caso de diversas medidas. 


\subsection{Para los estudiantes y de forma espontánea, la mejor representante de una serie de medidas no es la media}

También se ha visto que los tipos de razonamiento estadísticci resultan ajenos a los estudiantes de este nivel. $\mathrm{O}$, dicho de forma más precisa, incluso, si los conocen bajo una forma teórica, no son conscientes de que resultan relevantes para el proceso de medida. Los estudian. tes tienen que volver a meditar sobre los conceptos ligados a población, muestra, intervalo de seguriđad, etc., en el matco del proceso de datos. Por ejemplo, conocen perfectamente el concepto de media y el método para hallarla y, sin embargo, una encuesta anterior al inicio de las clases ha mostrado que solamente el $45 \%$ de los estudiantes consideró la media como la mejor representación de la serie de medidas, eligiendo algunos de ellos dicha media después de haber eliminado los valores máximo y mínimo. Otro $30 \%$ prefirió la moda y cl $25 \%$, la mediana.

\section{LAS OPORTUNIDADES, DURANTE LA SESION, DE APRENDER ANÁLISIS ESTA- DISTICO DE ERRORES Y DE SUPERAR LOS EQUIVOCOS CONCEPTUALES}

\subsection{Fase preliminar: aprendizaje de los conceptos estadísticos}

Dada su escasa familiarización con la estadística, antes de la sesión, proponemos a los estudiantes que estudien algunos conceptos como media, desviación estándar, distribución de Gauss, muestra, población, intervalo de seguridad, histograma. Con este objeto, tal como hemos dicho anteriormente, tienen que resolver dos ejercicios tomados de un libro de texto clásico (colección Schaum) [Spiegel, 1987] sobre estadística.

Por ejernplo, uno de los ejercicios sirve para entender el difícil concepto de distribución de medias de muestras de tamaño $\mathrm{N}$ extraídas de una población. Ello requiere del estudiante que compare una población de cuatro cifras con la distribución obtenida de las medias de muestras de tamaño $N=2$, de esta población. Éste es un concepto difícil, que demuestra que la media de la población y la media de la distribución de las diferentes muestras son iguales. Inversamente, las desviaciones estándar son inversamente proporcionales a la raíz de $N$. De esta manera resulta evidente la ventaja de tomar muestras amplias para mejorar la precisión del resultado extraído de un serie de medidas.

En el otro ejercicio propuesto a los estudiantes, se les pide que comparen el análisis de una serie de $N$ medidas para dos valores de $N$. El ejemplo se refiere al diámetro de una esfera. Se dan la media y la desviación estándar para $N=20$ medidas y para $N=40$ medidas. Se piden intervalos de seguridad para niveles de seguridad del $95 \%$ y del $68 \%$. Para compararlos, los estudiantes deben dibujar estos intervalos de seguridad sobre un eje horizontal.
Con este ejemplo, esperamos dejar claro que, cuando crece $N$, la media es más bien constante y que la dispersión de la medida evoluciona poco. Un argumento cualitativo es que, cuando crece $N$, el histograma se hace más y más simétrico y más y más suave, pero las amplitudes no varían. Inversamente, los intervalos de seguridad varían en proporción inversa a la raíz de $N$. Una de las razones es que la desviación estándar de la distribución de las medias de muestras de $N$ medidas, extraídas de una población dada, disminuye como $\sqrt{N}$. Dicho de otro modo, pasando del histograma de las medidas al histograma de las medias de las medidas tomado $N$ a $N$, se produce un cambio de $S_{N \cdot l}$ inversamente proporcional a la raíz de $N$.

En los párrafos siguientes, mostramos que la sesión que estamos estudiando permite entender visualmente algunas de estas difíciles nociones.

3.2. Visualización de histogramas de medidas: la desviación estándar fluctúa pero es invariante. La elección de la media

Tal como se ha dicho anteriormente, una condición para aplicar el análisis estadístico a una serie de medidas de la misma magnitud es la independencia. Éste es el caso que contemplamos, puesto que los estudiantes tienen dispositivos de medida nuevos e idénticos a su disposición. Consecuentemente, la comparación de los resultados obtenidos por una pareja de estudiantes con los de otra pareja resulta significativa. La comparación puede hacerse con los experimentos de los estudiantes el mismo día, así como durante las semanas previas. Los resultados de las parejas de estudiantes se acumulan semana tras semana hasta el final del semestre, en que se habrán obtenido unos 400 valores de $V_{0}$. Así, tenemos la posibilidad de un análisis estadístico de una muestra amplia, cuyo histograma se acercará a una población gaussiana.

Al final del propio trabajo experimental, cada pareja de estudiantes tendrá tres valores de $V_{o r}$ obtenidos de tres curvas $I=f\left(V_{G 2}\right)$. Introducirán estos valores mediante un programa de introducción de datos escrito en PASCAL, para un ordenador MICRAL. Los estudiantes podrán visualizar el histograma de cualquiera de los conjuntos de medidas:

- el conjunto de valores del grupo al que pertenecen; imprimirán el histograma con el fin de analizarlo;

- el conjunto de valores acumulados desde el principio del semestre.

Todo ello permite seguir las modificaciones del histograma a lo largo del periodo. El profesor imprime y expone el último histograma obtenido, que cada vez. tiene un aspecto más próximo al de una distribución de Gauss.

La figura 3 presenta los histogramas obtenidos para 34 , 108 y 289 medidas. Como puede verse, $V_{0}$ varía poco (los 
Figura 3

Algunos histogramas típicos obtenidos para $N=34,108$ y 289 medidas.

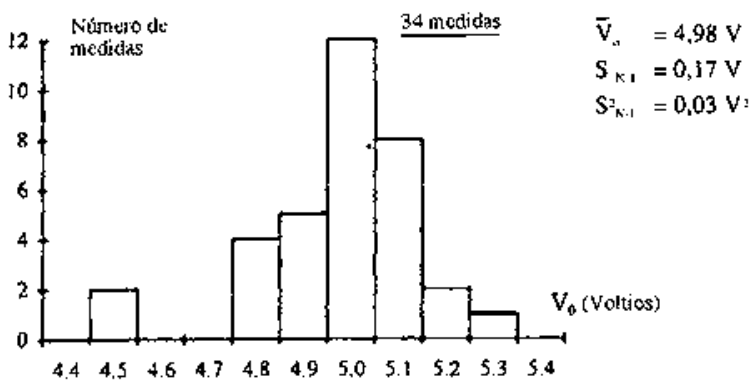

Intervalo de seguridad a $68 \%: 0,03 \mathrm{~V}$. Intervalo de seguridad a $95 \%: 0,06 \mathrm{~V}$.

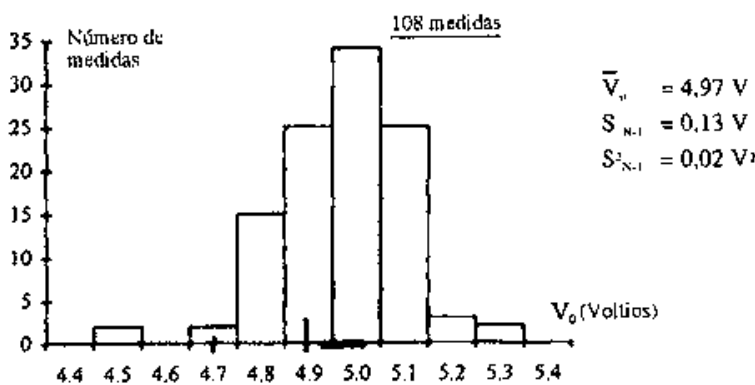

Intervalo de seguridad a $68 \%: 0,01 \mathrm{~V}$. Intervalo de seguridad a $95 \%: 0,03 \mathrm{~V}$.

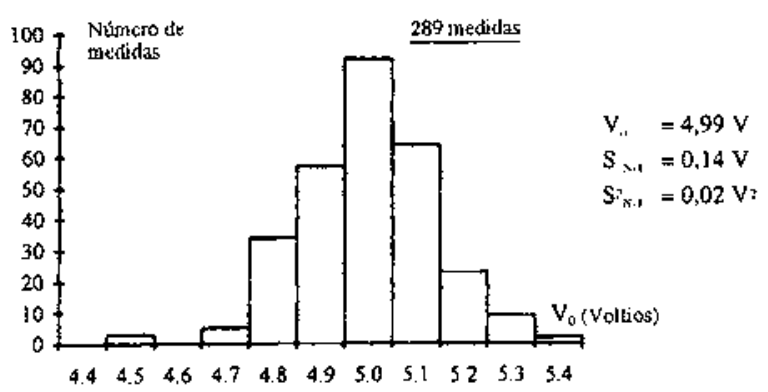

Intervalo de scguridad a $68 \%: 0,01 \mathrm{~V}$. Intervalo de seguridad a $95 \%: 0,02 \mathrm{~V}$.

valores respectivos son $4,98,4,97$ y 4,99 voltios), y ocurre lo mismo con $S_{\mathrm{N}-1}$ (los valores respectivos son $0,17,0,13$ y 0,14 voltios).

Por último, al final de la secuencia, el profesor imprime las curvas que representan la evolución de la media y de la desviación estándar con el número de medidas tomadas (Fig. 4). Éste parece ser un modo eficiente de verificar, cualitativa y visualmente, que la media resulta ser un valor representativo de la serie. La estabilidad de las dos cifras, en cuanto $N$ liega a 10 (razonable para un experimento en un laboratorio universitario), muestra que la dispersión de las medidas no varía mucho.
Figtura 4

Evolución de la media y de la desviación estándar con el número de medidas.
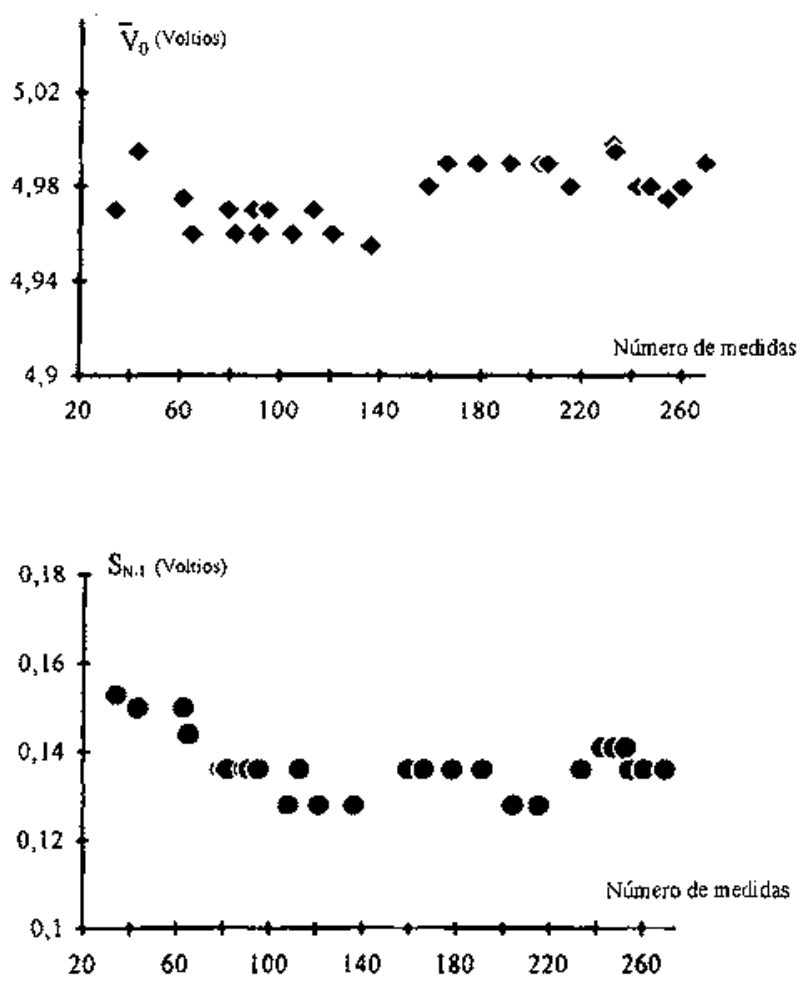

\subsection{Cálculo del intervalo de seguridad: aumentar el número de medidas mejora la precisión}

Con los 400 valores obtenidos de $V_{0}$, es posible realizar el análisis estadístico de una amplia muestra, cuyo hislograma se acerca a una población gaussiana. Los estu. diantes calculan el intervalo de seguridad asociado a las niveles de seguridad del $95 \%$ y del $68 \%$. Después, se hace la comparación entre los resultados obtenidos con cada método, clásico (una medida) y estadístico.

El tamaño del intervalo de seguridad para un mismo nivel de seguridad del $95 \%$, disminuye claramente cuando aumenta $N$ (los valores respectivos son $0^{\prime} 06,0^{\prime} 03$ y 0'02 voltios). Se pide a los estudiantes gue dibujen, sobre el eje horizontal del histograma de todo el grupo, el intervalo de seguridad correspondiente al $95 \%$, centrado en la media. (En la figura 3, se representa mediante un trazo grueso.) Con ello, utilizando los resultados del grupo con el que están trabajando, pueden entender que, independienternente de las calidades de medida concretas, la precisión mejora cuando se incrementa el número de medidas procesadas. 


\subsection{Varias medidas permiten evaluar cada conjunto de medidas: distinción entre precisión/exactitud, y errores aleatorios/sistemáticos}

También se pide a los estudiantes que comparen sus propios tres valores de $V_{0}$ con los resultados del grupo al que pertenecen. La figura 3 muestra tres de tales valores obtenidos por una pareja de estudiantes: $4,70 \mathrm{~V}, 4,90 \mathrm{~V}$ y $4,90 \mathrm{~V}$, que vienen representados por una línea vertical en el histograma $N=108$. Los dos estudiantes pueden hacer sus comentarios particulares acerca de sus resultados. En este caso, observaron que:

- sus tres valores son, todos ellos, menores que la media;

- sus tres valores están fuera del intervalo de seguridad para el $95 \%$.

Sus preguntas son:

- ¿Podrían nuestras medidas estar contaminadas por un pequeño error sistemático?

- ¿Merece la pena intentar verificar el instrumental para eliminarlo?

A pesar de ello, es posible que se den cuenta de que sus propias ntedidas estaban en un área de probabilidad relativamente alta, y podrían llegar a la conclusión de que, a pesar de haber realizado una serie de medidas, han sido incapaces, por sí mismos, de obtener el valor obtenido por la totalidad del grupo.

El resultado de tales comentarios personaies acerca de los propios resultados de los estudiantes les lleva a hacer una valoración de los conceptos de exactitud y de precisión. Les lleva a diferenciar estas dos cualidades, entendiendo que resulta inútil repetir y repetir las medidas para mejorar la precisión si la totalidad de las medidas está contaminada por un error sistemático. La incertidumbre sistemática es el límite de la precisión. Por tanto, idealmente, la elección de un número de medidas a realizar viene aconsejada por la evaluación de la incertidumbre sistemática, que es, a su vez, aconsejada por consideraciones físicas.

Los estudiantes pueden entender todo lo anterior al facilitarles el valor de la excitación potencial del mercurio, tomándolo de un manual: $4,89^{\mathrm{eV}} \mathrm{V}$. Evidentemente, en cada medida, existe un ligero error sistemático introducido por el instrumento, lo cual demuestra que es inútil repetir y repetir las medidas para alcanzar una precisión inejor que $0,1 \mathrm{~V}$, para $V_{0}$.

\section{CONCLUSIÓN}

Hemos presentado una forma original de utilizar un experimento clásico como trabajo de laboratorio, dirigido a estudiantes que empiezan en la universidad. EI experimento de Franck y Hertz resulta especialmente relevante para ayudar a los estudiantes a entender los distintos conceptos del análisis estadístico de errores. La razón es que las medidas de las distintas parejas de estudiantes son realmente independientes y proporcionan histogramas aprovechables.

Ésta es una innovación que es consecuencia de una investigación didáctica y estrictamente enfocada a solucionar los problemas descubiertos en la propia investigación. Creemos conveniente destacar lo siguiente:

- La sesión es realmente viable. Desde el primer año en que se aplicó, han sido aproximadamente 400 estudiantes los que han realizado el experimento cada año, en las mismas condiciones. Parece perfectamente adaptado al primero o al segundo año de universidad.

- Evidentemente, obtener datos durante el semestre y exponerlos constituye una fuente de interés para los estudiantes. Semana tras semana, se van dcsarrollando los histogramas expuestos y su evolución revela la verdad. Así, los estudiantes se detienen en el pasillo para seguir su evolución y hacer comentarios sobre la misma. La motivación resulia satisfactoria.

- A menudo, surgen comentarios verdaderamente personales sobre las medidas de los estudiantes, al compararlas con el histograma general. Con este método, en lugar de esperar el mismo resultado de cada una de las parejas de estudiantes, los profesores interinos no rechazan los resultados personales sino que piden estos comentarios personales. Se percibe una mejora respecto al trabajo «clásíco» de laboratorio.

- En general, el análisis que peđimos consigue que la distinción entre error aleatorio y error sistemático, y entre precision y exactitud se haga evidente. Naturalmente, mediante un solo experimento es imposible alcanzar el objetivo de proporcionar a los estudiantes la capacidad y la iniciativa para elegir entre los métodos de medida clásico y estadístico, para escoger el instrumento apropiado de medida y para escoger el número adecuado $N$ de medidas. Sin embargo, queda de manifiesto que, de una forma bastante simple y convincente, los estudiantes desarrollan una actitud personal hacia los datos. Ello es, en nuestra opinión, la base para una correcta aproximación a la experimentación.

\section{AGRADECIMIENTOS}

Agradecemos a Jean Benard el haber escrito los programas de introducción de datos. Jean lo hizo para numerosos experimentos, escribiendo programas o, lo que es más sacrificado, ayudando a los estudiantes del Departamento de Ciencia de Ordenadores a escribirlos con éxito.

\section{NOTA}

Este artículo ha sido traducido del inglés por J. Tortella. 


\section{REFERENCIAS BIBLIOGRÁFICAS}

FONDÈRE, F. (1993). Traitement statistique des mesures en DEUG. Evaluation d'une innovation auprès des enseignants. Working paper. DidaScO.

.JAECH, J.L. (1985). Statistical analysis of measurement errors. Nueva York: J. Wiley and sons.

SÉRÉ, M.G., LARCHER, C. y JOURNEAUX, R. (1993). Learning statistical analysis of measurements errors (first year of University). Int. J. Sci. Educ., 15, pp. 427-438.

[Artículo recibido en abril de 1996 y aceptado en mayo de 1997.]
SÉRÉ, M.G. y JOURNEAUX, R. (1995). Le traitement des mesures en TP de Physique de DEUG: une innovation à Orsay. Didaskalia, 6, pp. 165-177.

SPIEGEL, M.R. (1987). Théorie et applications de la statistique. París: McGraw Hill. 
